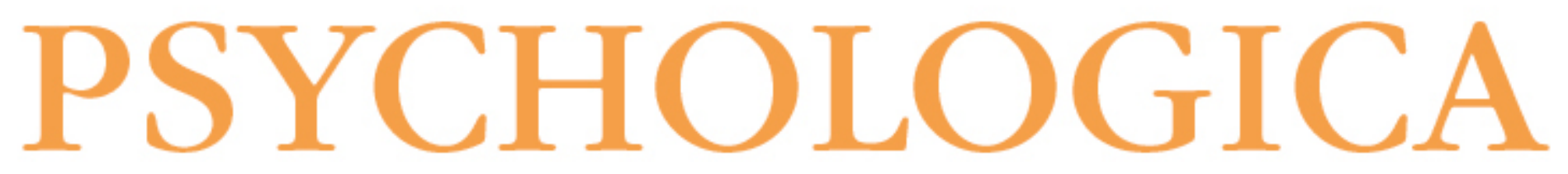

Influência da alexitimia nos processos atencionais: a deteção de expressões faciais emocionais
Autor(es):
Vitorino, Patrícia; Soares, Sandra C.; Cunha, Marina
Publicado por: Imprensa da Universidade de Coimbra
URL
persistente:
URI:http://hdl.handle.net/10316.2/29955
DOI:
DOI:http://dx.doi.org/10.14195/1647-8606_56_3
Accessed : $\quad$ 26-Apr-2023 05:37:22

A navegação consulta e descarregamento dos títulos inseridos nas Bibliotecas Digitais UC Digitalis, UC Pombalina e UC Impactum, pressupõem a aceitação plena e sem reservas dos Termos e Condições de Uso destas Bibliotecas Digitais, disponíveis em https://digitalis.uc.pt/pt-pt/termos.

Conforme exposto nos referidos Termos e Condições de Uso, o descarregamento de títulos de acesso restrito requer uma licença válida de autorização devendo o utilizador aceder ao(s) documento(s) a partir de um endereço de IP da instituição detentora da supramencionada licença.

Ao utilizador é apenas permitido o descarregamento para uso pessoal, pelo que o emprego do(s) título(s) descarregado(s) para outro fim, designadamente comercial, carece de autorização do respetivo autor ou editor da obra.

Na medida em que todas as obras da UC Digitalis se encontram protegidas pelo Código do Direito de Autor e Direitos Conexos e demais legislação aplicável, toda a cópia, parcial ou total, deste documento, nos casos em que é legalmente admitida, deverá conter ou fazer-se acompanhar por este aviso.

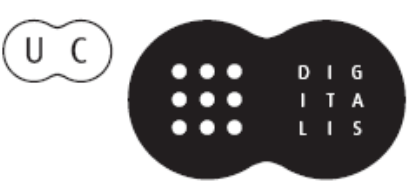


VOLUME 2013

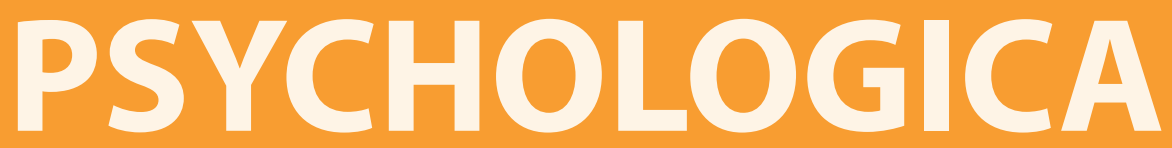

IMPRENSA DA UNIVERSIDADE DE COIMBRA

COIMBRA UNIVERSITY PRESS

FACULDADE DE PSICOLOGIA E DE CIÊNCIAS

DA EDUCAÇÃO DA UNIVERSIDADE DE COIMBRA 


\title{
Influência da alexitimia nos processos atencionais: A deteção de expressões faciais emocionais
}

\author{
Patrícia Vitorino ${ }^{1}$, Sandra C. Soares ${ }^{2}$ \\ e Marina Cunha ${ }^{3}$
}

The influnce of Alexithymia in the processes of attention: detection of emotional facial expressions

\begin{abstract}
Alexithymia is characterized by difficulties in emotional processing and facial expression recognition, essential processes in interpersonal relationship. In this study we intended to evaluate if facial expressions processing in individuals with high levels of alexithymia, especially negative emotional expressions (anger, disgust), is distinguished from the processing done by individuals with low levels of alexithymia. The participants (67 women) performed a visual search task in which sets of emotional facial expressions (happyness, anger and disgust) were presented among neutral facial expressions. The participants' task was to detect the presence or absence of these emotional facial expressions as quickly and accurately as possible. The results revealed no significant differences between the target groups. However, there was a differential processing of the emotional facial expressions according to the face gender. The negative emotional expressions on faces of men were promptly detected. On the other hand, the processing of faces of women who expressed anger led to a greater proportion of errors. The evolutionary assumptions associated with the processing of negative emotions, which the literature in the relationship between emotion and attention indicates as being an adaptive process in the general population,
\end{abstract}

1 Instituto Superior Miguel Torga. E-mail: patriciavvitorino@gmail.com

2 Universidade de Aveiro, Departamento de Educacação e IBILI, Faculdade de Medicina da Universidade de Coimbra

3 Instituto Superior Miguel Torga. 
may explain the absence of significant effects between the groups with high and low levels of alexithymia.

Key-words: alexithymia; visual search task; emotional detection

\section{Resumo}

A alexitimia carateriza-se pela dificuldade no processamento emocional e reconhecimento de expressões faciais, processos indispensáveis no relacionamento interpessoal. Neste estudo, pretendemos avaliar se o processamento de expressões faciais em indivíduos com altos níveis de alexitimia, sobretudo de expressões com cariz emocional negativo (raiva, nojo), é diferenciado do processamento efetuado por indivíduos com baixos níveis de alexitimia. Os participantes (67 mulheres) realizaram uma tarefa de procura visual onde se apresentavam conjuntos de expressões faciais emocionais (alegria, raiva, nojo), tendo os participantes de detetar de modo rápido e preciso a presença (ou ausência) destas expressões apresentadas entre expressões faciais neutras. Os resultados não revelaram quaisquer diferenças significativas entre os grupos. Contudo, verificou-se um processamento diferencial das expressões faciais emocionais em função do género da face. As expressões emocionais negativas em faces de homens foram mais rapidamente detetadas. Por outro lado, o processamento de faces de mulheres que expressavam raiva conduziu a uma maior proporção de erros. Os pressupostos evolutivos associados ao processamento das emoções negativas, que a literatura na área da relação entre a emoção e a atenção aponta como adaptativa na população em geral, poderá explicar a ausência de efeitos significativos entre o grupo com altos e baixos níveis de alexitimia.

Palavras-Chave: alexitimia; tarefa de procura visual; deteção emocional

\section{INTRODUÇÃO}

Em 1973, Sifneos propôs o conceito alexitimia para se referir aos indivíduos incapazes de identificar ou descrever adequadamente as suas emoções (Taylor, Ryan, \& Bagby, 1985). A alexitimia é um traço de personalidade estável que, segundo Nemiah, Freyberger e Sifneos (1976), consiste num modo menos simbólico (através de palavras ou imagens) de exprimir as emoções. Este traço de personalidade encontra-se presente em cerca de 10 a $20 \%$ da população geral (Taylor, Bagby, \& Parker, 1997), e caracteriza-se pela dificuldade em reconhecer e descrever emoções e sentimentos, diferenciar emoções e sentimentos de sensações corporais, pela 
diminuída capacidade para fantasiar e por um processamento cognitivo orientado para o exterior (Taylor et al., 1997).

Ao serem menos conscientes e sensíveis dos seus estados emocionais, os indivíduos com alexitimia tendem a apresentar maiores índices de psicopatologia (e.g., Taylor \& Bagby, 2004), apesar de ainda não ser claro se a alexitimia contribui para o desenvolvimento das perturbações ou se estas se desenvolvem na sua sequência (Silva \& Vasco, 2010).

Os estudos experimentais realizados com alexitímicos têm enfatizado a relação entre a alexitimia e a resposta emocional (e.g., Luminet, Rimé, Bagby, \& Taylor, 2004). As emoções conduzem a uma ativação fisiológica imediata, à qual o indivíduo atribui um significado em função do contexto em que a ativação ocorre, das experiências passadas e das memórias episódicas construídas (e.g., Sroufe, 1996). As emoções possuem, igualmente, uma função adaptativa (e.g., Lazarus, 1991; Scherer, 1984), permitindo estabelecer prioridades, tomar decisões e colocar ações em prática (Schwarz, 1990). Numa perspetiva evolutiva, as emoções orientam os nossos comportamentos em situações que se configuravam como relevantes para os nossos antepassados, nomeadamente, na obtenção de cuidados, proteção, recursos, encontro de amigos e construção de relações sociais (Öhman, 1986).

A investigação revela que as características dos indivíduos alexitímicos (e.g., dificuldades no relacionamento interpessoal, isolamento) estão associadas a enviesamentos cognitivos no processamento e regulação das emoções (Taylor et al., 1997). Concretamente, estes indivíduos apresentam um défice na avaliação do conteúdo de estímulos emocionais (e.g., Berthoz et al., 2002; Franz, Schaefer, Schneider, Sitte, \& Bachor, 2004), revelando dificuldades em transformar e codificar a informação emocional verbal [e.g., palavras relacionadas com emoções (e.g., Taylor et al., 1997)] e não-verbal [e.g., identificação de expressões faciais (e.g., Lane, Sechrest, Reidel, Weldon, Kaszniak, \& Schwartz., 1996)].

Estudos apontam que as dificuldades dos indivíduos com alexitimia não se focam apenas no processamento emocional interno, mas também no seu processamento interpessoal (perceção de emoções nos outros indivíduos) (e.g., Parker, Prkachin, \& Prkachin, 2005). A capacidade de detetar rápida e corretamente as expressões faciais emocionais, assim como a sua posterior identificação, constituem características essenciais a uma comunicação e interação social adequadas (e.g., Carton, Kessler, \& Pape, 1999). A comunicação do ponto de vista social tem um passado longínquo que remonta aos primórdios da nossa história evolutiva, dado que os nossos antepassados já comunicavam através de uma pré-linguagem (e.g., expressões faciais, fixação do olhar) (Darwin, 1998). A descodificação da informação emitida na comunicação (e.g., alteração das expressões faciais) permite que o indivíduo que recebe a mensagem se adapte e responda adequadamente 
às alterações ocorridas (e.g., Keltner, Ekman, Gonzaga, \& Beer, 2003), podendo desse modo controlar as reações emocionais na interação social, ajustar o seu comportamento evitando o conflito, e predizer as atitudes dos outros, atividades indispensáveis ao funcionamento social adequado (e.g., Mayer, DiPaolo, \& Salovey, 1990).

Estudos realizados na área do processamento emocional revelam que os indivíduos com elevados níveis de alexitimia são mais lentos (e.g., Prkachin \& Prkachin, 2001) e menos precisos na identificação de emoções em expressões faciais (e.g., Kano et al., 2003; Lane, Sechrest, Reidel, Shapiro, \& Kaszniak, 2000; Parker, Taylor, Bagby, \& Acklin, 1993), necessitando de mais recursos cognitivos para processar as emoções (Franz et al., 2004). Esta necessidade resulta do facto dos alexitímicos apresentarem representações menos detalhadas das expressões faciais (e.g., Reker et al., 2010), o que prejudica a quantidade e qualidade do seu relacionamento interpessoal (e.g., Mayer et al., 1990). Mais especificamente, estes indivíduos apresentam um comportamento frio, pouco assertivo e empático, e revelam dificuldade em compreender o ponto de vista dos outros e, consequentemente, em desenvolver relacionamentos íntimos (e.g., Humphreys, Wood, \& Parker, 2009).

As dificuldades apresentadas pelos indivíduos com elevados níveis de alexitimia na identificação de expressões faciais emocionais são apoiadas por resultados que indicam que a alexitimia está inversamente relacionada com a resposta automática de regiões do cérebro associadas ao processamento de expressões faciais emocionais (e.g., amígdala, hemisfério direito) (e.g., Moriguchi et al., 2006; Reker et al., 2010; Vermeulen, Luminet, Sousa, \& Campanella, 2008). Lane et al. (1996) constataram que a perceção consciente das emoções está associada ao aumento da atividade do córtex cingulado anterior (CCA) na população em geral (Blair, Morris, Frith, Perrett, \& Dolan, 1999). O CCA desempenha um papel crucial na avaliação da ativação emocional (Phan, Wager, Taylor, \& Liberzon, 2002), sendo que vários estudos concluíram que a alexitimia pode resultar da diminuição da ativação desta região, o que dificulta a comunicação entre o sistema límbico e o neocórtex (e.g., Mériau et al., 2006). Esta falha de comunicação prejudica assim a capacidade destes indivíduos identificarem e comunicarem o seu estado emocional (e.g., Kano et al., 2003; Mériau et al., 2006). Alguns estudos, com indivíduos com elevados níveis de alexitimia, apontam para um aumento da atividade do CCA em resposta a imagens positivas, mas diminuição da mesma perante imagens negativas, especialmente no processamento da raiva, o que afeta o reconhecimento explícito desta emoção em expressões faciais (e.g., Berthoz et al., 2002; Kano et al., 2003; Vermeulen, Luminet, \& Corneille, 2006; Vermeulen et al., 2008).

Numa perspetiva evolutiva, a raiva possui uma função adaptativa, apresentando um elevado valor de sobrevivência (e.g., Öhman, 1986). Há milhares de anos, a 
raiva permitia alertar o indivíduo para o perigo do ataque do inimigo, ativando-o para contra-atacar e eliminar o perigo (e.g., Izard, 1991). Atualmente esta emoção está relacionada com uma resposta a situações desagradáveis (interpretadas como ameaçadoras, ofensivas ou prejudiciais) que ativam e impulsionam o indivíduo para a ação (e.g., Izard, 1991). Assim, verificamos que o défice no processamento desta emoção pode comprometer a adaptação dos alexitímicos a estímulos ameaçadores e altamente ativadores do ponto de vista fisiológico, por exigir rápidas respostas de autorregulação que permitam lidar com exigências comportamentais futuras (e.g., Vermeulen et al., 2008).

Apesar de a literatura assinalar a importância de uma correta deteção e posterior identificação emocional na comunicação e interação sociais (Vanheule, Vandenbergen, Verhaeghe, \& Desmet, 2010), são poucos os estudos que analisaram a capacidade de deteção emocional nos alexitímicos. Um desses estudos (Vermeulen et al., 2008) avaliou a forma como os indivíduos com elevados níveis de alexitimia categorizam as emoções negativas (e.g., raiva) conforme estas vão sofrendo alterações (morphed faces) até se transformarem numa outra emoção distinta (e.g., desgosto), e verificou que os indivíduos apresentavam atrasos na discriminação emocional, que se iniciavam ao nível da atenção. Por outro lado, alguns estudos indicam que a alexitimia não está relacionada com os processos atencionais, tais como a deteção da relevância dos estímulos emocionais, mas sim com processos posteriores, como o reconhecimento de expressões faciais (e.g., Vermeulen et al., 2006).

Como podemos verificar, têm sido realizados vários estudos que apontam que os alexitímicos apresentam dificuldades ao nível do processamento emocional (e.g., Kano et al., 2003). Porém, como mencionámos, alguns resultados são contraditórios (e.g., Vermeulen et al., 2006). Esta inconsistência poder ser explicada pelo recurso a diferentes medidas comportamentais (e.g., Jessimer \& Markham, 1997), neuro-imagem (e.g., Kano et al., 2003; Kugel et al., 2008), utilização de diferentes tarefas como faces transformadas, em que a emoção da expressão facial se vai alterando ao longo das diferentes imagens até se transformar numa nova emoção (e.g., Mériau et al., 2006), chimeric tasks, em que cada metade da face representa uma emoção diferente (e.g., Jessimer \& Markham, 1997), faces mascaradas, em que a emoção é mascarada por outras emoções (e.g., Kugel et al., 2008). São utilizados métodos variados como, pedir aos participantes para identificarem as diferentes emoções (e.g., Kugel et al., 2008), a intensidade de cada emoção (e.g., Kano et al., 2003) ou o sexo das pessoas fotografadas a expressarem diferentes emoções (e.g., Kano et al., 2003). Adicionalmente, são utilizados diversos estímulos, nomeadamente, verbais (e.g., Lane et al., 2000, tarefa 2) não-verbais (e.g., Lane et al., 2000, tarefa 4) e mistos (e.g., Vermeulen et al., 2006). Diferem igualmente quanto ao sexo dos 
participantes, sendo que uns utilizam apenas homens (Berthoz et al., 2002), outros apenas mulheres (e.g., Reker et al., 2010), e ainda outros, ambos os sexos (Vermeulen et al., 2006). Por último apresentam variações no tamanho da amostra, existindo uns estudos que utilizaram muitos (e.g., Vermeulen et al., 2008) e outros poucos participantes (e.g., Berthoz et al., 2002).

No presente estudo, para avaliar a capacidade de deteção emocional dos indivíduos com elevados níveis de alexitimia, optámos por uma das medidas mais utilizadas no estudo da relação entre a atenção e a emoção, a tarefa de procura visual (Pashler, Dobkins, \& Huang, 2004). A relevância desta tarefa deve-se ao facto de permitir captar aspetos importantes do nosso mundo visual (e.g., procurar estímulos emocionais entre neutros, que nos permitam agir de forma rápida e adequada), sendo uma ferramenta eficaz na investigação da atenção seletiva, ao permitir-nos selecionar uma informação em detrimento de outra quando confrontados com a apresentação simultânea de diferentes categorias de informação visual (e.g., Driver \& Frackowiak, 2001). Neste tipo de tarefa é pedido aos participantes que detetem da forma mais rápida e precisa um alvo discrepante (item ou imagem de uma categoria diferente de todas as outras) entre vários distratores (i.e., itens ou imagens com as mesmas características ou pertencente à mesma categoria), permitindo-nos avaliar os tempos de resposta bem como a precisão das respostas dadas.

São diversos os estímulos (de cariz emocional ou não) utilizados nas tarefas de procura visual (Galera, 2003). A utilização de expressões faciais pertencentes a diferentes categorias emocionais, sobretudo as negativas, permite verificar se a evolução dos sistemas de defesa humana está associada à deteção de ameaças num ambiente (Hansen \& Hansen, 1988). Nesse sentido, no presente estudo utilizámos estímulos emocionais (expressões faciais) positivos, negativos e neutros para perceber como a atenção interage com o processamento de emoções nos indivíduos com elevados níveis de alexitimia, comparativamente com indivíduos com baixos níveis de alexitimia. A relevância desta análise prende-se com o facto de as emoções possuírem um papel fundamental na orientação de recursos de atenção para os estímulos importantes (e.g., Oatley \& Jenkins, 1996) que, neste caso particular, poderá ter importantes repercussões ao nível da interação social (e.g., Carton et al., 1999).

Neste estudo pretendemos obter uma amostra representativa de indivíduos com elevados níveis de alexitimia que nos permita perceber se os processos atencionais são afetados durante o processamento de emoções (expressões faciais) positivas, negativas e neutras, isto é, se o défice existente no processamento emocional dos indivíduos com elevados níveis de alexitimia se inicia ao nível dos processos atencionais ou apenas posteriormente (no reconhecimento emocional). Nesse sentido, e de acordo com os resultados obtidos ao nível da capacidade de deteção e 
identificação emocional dos indivíduos com elevados níveis de alexitimia (apesar da utilização de tarefas díspares e diferentes da tarefa de procura visual), pressupomos que este grupo de indivíduos poderá apresentar maior dificuldade (maiores tempos de reação e menor precisão nas respostas) em detetar expressões faciais de emoções negativas. Adicionalmente, considerando que estudos anteriores indicam que os indivíduos com elevados níveis de alexitimia apresentam uma diminuição da atividade do CCA (e.g., Vermeulen et al., 2006), estrutura envolvida no processamento da raiva (e.g., Blair et al., 1999), pretendemos avaliar se estes indivíduos são mais lentos e menos precisos na deteção de faces que exibem essa expressão emocional específica (i.e., raiva).

\section{METODOLOGIA}

\section{Participantes}

Dos 145 individuos que participaram no nosso estudo, foram selecionados 67 para constituir a nossa amostra, consoante a pontuação obtida na versão portuguesa da Toronto Alexithymia Scale de 20 itens (Bagby, Parker, \& Taylor, 1994a). Recorremos a este instrumento de avaliação por ser o mais comumente utilizado na autoavaliação da alexitimia (e.g., Mantani, Okamoto, Shirao, Okada, \& Yamawaki, 2005) e se encontrar validado para a população portuguesa, apresentando boa consistência interna, boa validade convergente e divergente e confiabilidade teste-reteste (Prazeres, Parker, \& Taylor, 2000; Veríssimo, 2001).

Assim, e de acordo com os níveis de alexitimia apresentados, os 67 participantes foram divididos em dois grupos, o experimental (altos níveis de alexitimia) e o de controlo (baixos níveis de alexitimia). Porém, dada a dificuldade em obter uma amostra de alexitímicos significativa, por esta apenas afetar 10 a $20 \%$ da população geral (Taylor et al., 1997), surgiu a necessidade de alterar os pontos de corte estabelecidos para o grupo clínico $(\mathrm{x} \geq 61$ ), alargando a amostra ao grupo subclínico $(61 \geq x \geq 52)$. Deste modo, foram determinados pontos de corte para baixos níveis de alexitimia $(x \leq 45)$ e elevados níveis de alexitimia $(x \geq 52)$, sendo o grupo de controlo constituído por 34 participantes $(\mathrm{M}=37.2$, DP $=5.4)$ e o experimental por 33 participantes $(\mathrm{M}=59.6, \mathrm{DP}=6.6)$.

De modo consistente com alguns estudos com indivíduos com elevados níveis de alexitimia que utilizam apenas mulheres (e.g., Karlsson, Näätänen, \& Stenman, 
2008; Mériau et al., 2006; Reker et al., 2010), uma vez que os homens e as mulheres processam os estímulos emocionais de modo diferente (e.g., as mulheres relatam reações emocionais mais intensas que os homens, quando avaliam expressões faciais (e.g. Chentsova-Dutton \& Tsai, 2007; Mériau et al., 2006), a nossa amostra foi constituída, exclusivamente, por participantes do sexo feminino.

Os participantes tinham idades compreendidas entre os 18 e os 35 anos $(\mathrm{M}=$ 24.4, DP $=4.2$ ) e frequentavam $(73.1 \%)$ ou haviam frequentado (26.9\%) o ensino superior. Os participantes que se encontravam a frequentar o ensino superior foram recrutados de 18 cursos diferentes (e.g., Psicologia, Serviço Social), estando a maioria a frequentar o primeiro ciclo de estudos. Os participantes dos diferentes cursos eram oriundos de diferentes institutos superiores de várias cidades do País (Algarve, Aveiro, Beja, Coimbra, Évora, Leiria, Lisboa), permitindo-nos assim obter uma amostra com maior representatividade.

\section{Instrumentos}

O nosso estudo passou por duas fases de recolha de dados distintas. Na primeira fase, aplicou-se a escala de alexitimia de Toronto, de 20 itens, em formato de papel e caneta, que nos permitiu proceder à divisão da nossa amostra pelos grupos experimental e de controlo.

Na segunda fase de recolha de dados, recorremos a uma tarefa comportamental (procura visual), realizada em computador, para avaliar a influência da alexitimia nos processos atencionais, isto é, a capacidade (tempo e precisão da resposta) dos indivíduos com elevados níveis de alexitimia detetarem expressões faciais emocionais.

Os instrumentos de recolha de dados encontram-se seguidamente descritos pela ordem corresponde à sequência em que os mesmos foram administrados.

\section{Escala de alexitimia de Toronto de 20 itens (TAS-20)}

Recorremos à escala de avaliação da alexitimia, o TAS-20 (Toronto Alexithymia Scale-20; Bagby et al., 1994a) para proceder à seleção da amostra, dado este ser o instrumento mais utilizado na sua autoavaliação, apresentando uma adequada precisão e validade para a avaliação deste constructo (Prazeres et al., 2000). A última versão desta escala, a TAS-20, resulta de um trabalho continuado por parte dos seus autores para aperfeiçoar as suas propriedades psicométricas, representando uma melhoria significativa face às versões anteriores, TAS e TAS-R (Bagby et al., 1994a; Bagby, Parker, \& Taylor, 1994b). Este instrumento foi traduzido e adaptado 
para a população portuguesa por Prazeres et al. (2000), mantendo-se a sua estrutura fatorial original, tal como a coerência interna (alfa de Cronbach de 0.75) e a precisão teste-reteste (num intervalo de 3 a 6 semanas) (Veríssimo, 2001).

Tal como na versão original, a escala apresenta pontos de corte para o grupo clínico (pontuações superiores a 60) e para a população normal (pontuações inferiores a 52), correspondendo os valores intermédios a índices de alexitimia moderada (grupo subclínico) (Taylor et al., 1997).

A TAS é constituída por 20 itens, onde é solicitado ao indivíduo que registe o seu grau de concordância relativamente a cada um dos itens, recorrendo a uma escala do tipo Lickert de cinco pontos, que varia entre 1 (desacordo total) e 5 (acordo total). Estes permitem obter uma pontuação global da alexitimia (entre 20 e 100), bem como dos três fatores que nos dão acesso aos componentes mais representativos do constructo, designadamente, F1 que corresponde à dificuldade em identificar emoções e em distingui-las das sensações corporais da emoção (e.g., "Muitas vezes sinto-me confuso(a) em relação ao tipo de emoção que estou a sentir"), F2 que se refere à dificuldade em descrever as emoções aos outros (e.g., "É-me difícil encontrar as palavras certas para descrever os meus sentimentos"), e F3 que corresponde ao pensamento orientado para o exterior (e.g., "Prefiro mais falar com as pessoas sobre o seu dia-a-dia do que sobre os seus sentimentos") (Taylor et al., 1997). Porém, no presente estudo utilizamos apenas os resultados globais de modo a diferenciarmos o grupo experimental (elevados níveis de alexitimia) do grupo de controlo (baixos níveis de alexitimia).

\section{Medidas comportamentais: Tarefa de procura visual}

Todos os participantes selecionados para a segunda fase de recolha de dados, executaram uma tarefa de procura visual, adaptada de uma das utilizadas nas experiências de Öhman, Juth, \& Lundqvist (2010). Na tarefa foram utilizadas fotografias de faces reais para assegurar a validade ecológica da tarefa (Öhman et al., 2010). Nesse sentido, recorremos a fotografias de faces de homens (16) e de mulheres (16) com diferentes expressões emocionais, selecionadas a partir da base de dados "Karolinska Directed Emotional Faces" (Lundqvist, Flykt, \& Öhman, 1998) e validadas por Goeleven, Raedt, Leyman, e Verschuere (2008). Nesta tarefa pretendíamos verificar se a diferenciação de categorias de faces emocionais (especialmente a raiva) era influenciada pelos níveis de alexitimia apresentados pelos indivíduos.

As faces foram apresentadas aos participantes (144 conjuntos de seis imagens em cada matriz de imagens), organizadas em forma de círculo e distando entre 
si de modo equidistante (ver Figura 1). As imagens que compunham cada matriz eram todas do mesmo indivíduo (matriz homogénea - só faces de um homem ou só faces de uma mulher), com todas as faces a apresentarem uma orientação frontal (ver Figura 1), podendo a sua expressão variar entre expressões neutras, com raiva, nojo e alegria. Em metade dos ensaios foram apenas apresentadas matrizes com faces neutras, enquanto nos restantes $50 \%$ dos ensaios, uma face neutra foi substituída por um alvo (face) emocional discrepante (raiva, nojo, ou alegria). Foram usados 3 blocos de imagens (de 48 ensaios cada), sendo a sua ordem de apresentação contrabalanceada. Para além disso, foi igualmente contrabalanceada a ordem de apresentação de faces de homens e mulheres apresentadas entre cada matriz.

A seleção de emoções positivas (alegria) e negativas (raiva e nojo) prendeu-se com o facto de estudos anteriores sugerirem que, nos indivíduos com elevados níveis de alexitimia, o processamento de expressões positivas difere do de expressões negativas (e.g., Adolphs, Damasio, Tranel, \& Damasio, 1996), apresentando estes indivíduos piores resultados na identificação de emoções negativas (e.g., Berthoz et al., 2002; Kano et al., 2003; Vermeulen et al., 2006). Para além disso, e dado que a literatura aponta para uma maior consistência nas variações específicas no processamento da raiva em alexitímicos, pretendemos incluir uma segunda expressão igualmente negativa (nojo), que geralmente associa, tal como a raiva, uma elevada ativação fisiológica, assim como elevados níveis de desagradabilidade (Goeleven et al., 2008).

Os ensaios foram iniciados pelo aparecimento da cruz de fixação no centro do monitor, que se manteve um segundo, sendo posteriormente substituída por matrizes constituídas por fotografias de faces que se mantiveram, sem limite de tempo, até ser dada uma resposta pelo participante. Os participantes encontravam-se sentados a 70 centímetros do monitor, sensivelmente, registando as suas respostas através de teclas do computador selecionadas para o efeito. Assim, se entre as faces neutras surgia uma face com uma expressão facial discrepante (raiva, nojo ou alegria), os participantes deveriam pressionar a tecla " $M$ " e, caso todas as faces apresentassem uma expressão emocional neutra, deveriam pressionar a tecla " $Z$ ”. Três segundos após pressionarem numa das teclas o computador avançava automaticamente para um novo ponto de fixação, sendo posteriormente apresentado um novo conjunto de imagens, e assim sucessivamente.

A tarefa foi executada num computador (15 polegadas), com recurso ao software E-Prime 2.0 (Schneider, Eschman, \& Zuccolotto, 2002). Os dados foram recolhidos individualmente ou em grupos de dois, num gabinete calmo e apropriado para o efeito. 


\section{Procedimento}

Os participantes deste estudo foram submetidos a dois procedimentos distintos, um questionário e uma tarefa de procura visual, que tiveram, sensivelmente, uma duração de 10 e 15 minutos por participante, respetivamente.

Antes de iniciar a recolha de dados, foi obtido o consentimento informado junto dos participantes, no qual lhes era assegurado que a informação recolhida ao longo do estudo seria confidencial e utilizada apenas para fins de investigação, sendo-lhes facultado o direito de desistir do mesmo a qualquer momento. Foram recolhidos dados pessoais como o nome, contacto eletrónico e/ou telefónico, com o objetivo único de entrar em contacto com os participantes caso estes fossem selecionados para a segunda fase do estudo (tarefa de procura visual).

Aquando da realização da tarefa de procura visual, pediu-se aos participantes que se sentassem confortavelmente. Esta tarefa foi precedida de itens de treino e das respetivas instruções (em computador) seguidamente apresentadas:

Bem-vinda! Esta experiência é composta por várias fases. Nesta tarefa vai ver uma série de imagens. As imagens são fotografias de diferentes indivíduos com diferentes expressões emocionais (neutras, alegres, raiva e nojo). Em cada apresentação aparecem 6 fotografias, dispostas em círculo. Algumas vezes todas as expressões faciais são iguais, ou seja, são todas neutras. Outras vezes aparece uma expressão diferente das outras. Por exemplo podem ser 5 expressões neutras e uma de nojo, ou então, por exemplo ser uma expressão alegre no meio de expressões neutras. A sua tarefa é decidir tão rapidamente quanto possível, se todas as imagens são da mesma categoria (expressões faciais neutras), ou então, se uma é diferente das outras. Se todas as imagens forem da mesma categoria, prima a tecla “Todas Iguais". Se apenas uma expressão facial de raiva, alegria ou nojo, no meio de expressões todas neutras, prima a tecla "Uma diferente". Antes de cada ciclo de imagens, aparece uma cruz no meio do monitor. Quando a cruz aparecer, deve fixar o olhar nela. Quando a cruz desaparecer e as imagens forem apresentadas, pode olhar para onde e como quiser. Lembre-se que deve responder o mais rápido que puder. Se acontecer carregar numa tecla errada, não se preocupe, é só continuar. Tente manter a concentração durante toda a série de imagens. Antes da experiência começar vai ver alguns exemplos. Depois dos exemplos de treino vai ter a possibilidade de fazer uma pequena pausa antes de começar a experiência.

As instruções foram repetidas após o intervalo existente entre cada bloco de imagens. No final da tarefa, agradeceu-se aos participantes pela sua colaboração no estudo. 


\section{Análises estatísticas}

As análises estatísticas necessárias ao tratamento de dados foram efetuadas com recurso ao Statistical Package for the Social Sciences (SPSS), versão 19 , sendo o nível de significância estatístico fixado em $\mathrm{p}<.05$ para todas as comparações.

Nos dados recolhidos na tarefa de procura visual, foram realizadas análises de variância de medidas repetidas (ANOVA), que incluíam um fator inter-sujeito [tipo de grupo (elevados valores de alexitimia vs. baixos valores de alexitimia)] e dois fatores intra-sujeitos [alvo (neutro ou ausente, raiva, nojo, alegria) e género associado à face (feminino vs. masculino)]. Foram ainda realizadas comparações post hoc (com recurso ao teste Tukey HSD) e averiguados outros resultados significativos através de análises estatísticas específicas.

Os tempos de resposta (TR) médios e as proporções de respostas corretas (i.e., pressionar a tecla correspondente à presença de um alvo quando efetivamente a matriz de imagens inclua um alvo - expressão emocional, e vice-versa) foram calculados para cada participante e para cada tipo de alvo. As respostas incorretas foram excluídas das análises. Os TR inferiores e superiores a 3 desvios-padrão, calculados separadamente para cada indivíduo, foram substituídos pela média individual de \pm 3 desvios-padrão.

\section{RESULTADOS}

Análise dos dados da tarefa de procura visual

\section{Tempo de resposta (TR)}

Contrariamente ao esperado, não foram obtidos quaisquer efeitos significativos envolvendo a variável grupo (ver Figura 2). Os resultados não revelaram diferenças gerais no processamento atencional entre o grupo com altos e baixos níveis de alexitimia $(M=1421, D P=400$ e $M=1480, D P=400$, respetivamente), $F(1,65)=$ $.36, p>.05, \eta^{2}=.01$. Para além disso, não foram verificadas diferenças entre os grupos ao nível da deteção das diferentes expressões emocionais, $F(3,195)=1.84$, $p>.05, \eta^{2}=.03$, em função do género da face apresentada nas matrizes, $F(1,65)$ 
$=.01, p>.05, \eta^{2}=.00$, nem na interação entre estes três fatores (i.e., grupo, alvo, e género), $F(3,195)=.56, p>.05, \eta^{2}=.01$.

No geral, os resultados revelaram TR mais lentos perante a ausência $(M=1865$, $D P=611$ ) do que perante a presença de alvos nas matrizes (raiva: $M=1328, D P$ = 363; nojo: $M=1285, D P=264$; e alegria: $M=1325, D P=363$ ), expresso através do efeito principal do tipo de alvo, $F(3,195)=1.84, p<.0001, \eta^{2}=.03$. Análises à posteriori, usando o teste Tukey, revelaram não existir diferenças na deteção dos diferentes tipos de expressão emocional ( $p s>.05$ ). Contudo, a deteção dos alvos emocionais parece ter sido modelada em função do género da face apresentada nas matrizes, uma vez que uma interação significativa entre o alvo e o género, $F(3,195)$ $=41.13, p<.0001, \eta^{2}=.39$, revelou TR mais rápidos na deteção de alvos emocionais negativos (raiva e nojo) para faces de homens (raiva: $M=1226, D P=299$; nojo: $M$ $=1166, D P=324)$, comparativamente com as condições em que as faces apresentadas eram de mulheres (raiva: $M=1429, D P=443$; nojo: $M=1405, D P=348$ ) ( $p s$ $<.0001$ ) (ver Figura 3). Para expressões emocionais de alegria os resultados não revelaram diferenças em função do género da face apresentada $(M=1350, D P=$ 366; e $M=1301, D P=376$, para faces de homens e mulheres, respetivamente [ver Figura 3]). Verificou-se ainda que, no geral, os participantes eram mais rápidos a detetar expressões faciais de homens $(M=1389, D P=45.20)$ do que expressões faciais de mulheres $(M=1512, D P=434)$, o que foi demonstrado através de um efeito principal de género, $F(1,65)=86.88, p<.0001, \Pi^{2}=.57$.

\section{Proporção de respostas corretas}

Os resultados obtidos através da análise de proporção de respostas corretas foram consistentes com os dos TR (ver Figura 4). Não foi observado qualquer efeito significativo envolvendo a variável grupo (ver Figura 4), ou seja, os indivíduos com elevados níveis de alexitimia não diferiram do grupo de controlo no processamento atencional geral $(M=0.95, D P=.04 ; M=0.96, D P=.04$, para o grupo experimental e de controlo, respetivamente), $F(1,65)=2.68, p>.05, \eta^{2}=.04$, assim como na deteção dos diferentes tipos de alvo emocionais, $F(3,195)=2.02, p>.05, \eta^{2}=.03$. Para além disso, a deteção dos alvos entre os dois grupos não variou em função do género da face apresentada, $F(1,65)=.04 ; p>.05, \eta^{2}=.001$.

A análise da proporção de respostas corretas demonstrou que ambos os grupos produziram maior proporção de respostas corretas quando não existia um alvo na matriz $(M=0.99 ; D P=.04)$, comparativamente com as condições em que se verificava a presença de um alvo (raiva: $M=0.91 ; D P=.08$; nojo: $M=0.95 ; D P=$ .06 ; e alegria: $M=0.96 ; D P=.04), F(3,195)=25.13, p<.0001, \eta^{2}=.28$. Análises post hoc (tukey HSD), permitiram verificar diferenças significativas entre a deteção 
de faces de raiva e a deteção das restantes expressões faciais emocionais (nojo e alegria) ( $p s<.01)$ (ver Figura 5). Adicionalmente, verificámos que os participantes acertavam mais nas matrizes que incluíam faces de homens $(M=0,97 ; D P=.03)$, comparativamente com matrizes com faces de mulheres $(M=0,94 ; D P=.05)$, como foi evidenciado através do efeito principal de género, $F(1,65)=26.84, p<.0001$, $\eta^{2}=.29$. Este efeito era modulado em função do tipo de alvo, uma vez que a menor proporção de respostas corretas para as expressões de raiva era particularmente evidente quando as faces apresentadas na matriz eram femininas $(p s<.0001), F$ $(3,195)=25.99, p<.0001, \eta^{2}=.29$.

\section{DISCUSSÃO}

A presente investigação objetivava avaliar o processamento emocional de expressões faciais em indivíduos com elevados níveis de alexitimia face aos indivíduos com baixos níveis de alexitimia. Nesse sentido, pretendíamos perceber se os processos atencionais eram afetados durante o processamento de emoções (expressões faciais) positivas, negativas e neutras, isto é, se a dificuldade existente no processamento emocional dos indivíduos com elevados níveis de alexitimia se inicia logo nos processos de deteção emocional, o que se refletiria em TR mais elevados e menor proporção de respostas corretas na deteção de faces com expressões emocionais negativas.

Como referimos anteriormente, a capacidade de detetar expressões faciais é indispensável ao relacionamento interpessoal dado permitir a regulação entre o emissor e o recetor, ao evocar respostas automáticas, suscetíveis de influenciar as respostas comportamentais subsequentes (e.g., Keltner et al., 2003; Prkachin, Casey, \& Prkachin, 2009). A deteção de uma emoção deve permitir que uma pessoa avalie rapidamente a decisão a tomar, sendo o tempo que esta demora a ser detetada um bom indicador da capacidade de adaptação do indivíduo (e.g., Vermeulen et al., 2008). Vários estudos apontam para um défice no processamento emocional dos indivíduos com elevados níveis de alexitimia (e.g., Kano et al., 2003), que afetam a capacidade de alocar os recursos atencionais na deteção dos estímulos salientes (e.g., Vermeulen et al., 2008). Nesse sentido, estes indivíduos necessitam de mais recursos cognitivos para processar as emoções (e.g., Aftanas \& Varlamov, 2007; Franz et al., 2004), sendo mais lentos (e.g., Prkachin \& Prkachin, 2001) e menos precisos na identificação de emoções em expressões faciais (e.g., Kano et al., 2003; Lane et al., 2000; Parker et al., 1993). Contrariamente, outros estudos indicam que a alexitimia não está relacionada com os processos aten- 
cionais, como a deteção da relevância dos estímulos emocionais, mas sim com processos posteriores, envolvidos na capacidade de identificação emocional (e.g., Vermeulen et al., 2006).

Os resultados obtidos no nosso estudo indicam que o TR e a precisão da deteção emocional não diferem significativamente entre os participantes com baixos e elevados níveis de alexitimia. Estas inconsistências entre os estudos, provavelmente, devem-se ao facto de a dificuldade dos indivíduos com elevados níveis de alexitimia detetarem as emoções (negativas) apenas se verificar em janelas temporais específicas (Parker et al., 2005). Nesse sentido, o facto de não ter existido uma manipulação do tempo de exposição das matrizes, que nos permitisse avaliar o curso temporal da deteção das diferentes expressões faciais, poderá ter diluído a diferenciação entre os dois grupos. Adicionalmente, é importante referir que os resultados obtidos nas expressões faciais neutras, de alegria, e de nojo, quanto à proporção de respostas corretas, sugerem a ocorrência de um efeito teto ao nível do desempenho dos participantes (i.e., o teste é demasiado fácil, sendo obtidos resultados muito próximos do resultado máximo, não permitindo distinguir ambos os grupos quanto à sua pontuação [Stratton \& Hayes, 1999]). Este efeito provavelmente deve-se ao facto de os distratores serem homogéneos (i.e., apenas faces de expressão neutra), podendo ser facilmente agrupados, aumentando a saliência do alvo emocional e, consequentemente, facilitando a sua deteção (e.g., Rauschenberger \& Yantis, 2006; Wolfe, 1998).

Vários estudos no âmbito do processamento emocional com indivíduos que apresentam elevados níveis de alexitimia (e.g., Vermeulen et al., 2008) apontam para maiores dificuldades (TR mais elevados e menor proporção de respostas corretas) no processamento de emoções negativas, especialmente no processamento da raiva (e.g., Kano et al., 2003). Vermeulen et al. (2008) sugerem que o atraso na capacidade de discriminar esta emoção (TR mais elevados), ocorre desde o seu processamento atencional. Contudo, no nosso estudo, as diferenças ao nível do TR e precisão da deteção desta emoção não foram significativas entre os dois grupos. Isto é, apesar de os participantes com elevados níveis de alexitimia apresentarem um défice no processamento da raiva perante alvos de mulheres, este efeito mantém-se nos participantes com baixos níveis de alexitimia, o que nos alerta para a possibilidade de o défice na capacidade de processamento da raiva não ser exclusivo dos alexitímicos, mas comum a toda a população.

Apesar de os objetivos desta investigação se centrarem nos resultados obtidos ao nível da variável grupo, é importante salientar a obtenção de resultados gerais significativos, que são relevantes no âmbito da literatura que analisa a relação entre a atenção e emoção. A maioria dos estudos que exploraram a relação entre emoção e atenção (com recurso a faces esquemáticas e reais) demonstram que uma 
expressão facial de ameaça (raiva) envolverá o direcionamento mais rápido e eficaz de recursos atencionais (e.g., Öhman, Lundqvist, \& Esteves, 2001), sendo os indivíduos mais rápidos e precisos a detetar esta emoção. Do ponto de vista evolutivo, a raiva apresenta impactos diferentes em ambos os sexos, por os homens terem de correr mais riscos devido à concorrência intrassexual (macho-macho), enquanto as mulheres correm riscos essencialmente no domínio da reprodução e criação dos filhos (Fessler, Pillsworth, \& Flamson, 2004), estando menos predispostas a agir agressivamente em situações de risco. Esta predição baseou-se no facto do risco de ser superado por um rival ter, provavelmente, exigido uma maior aptidão na utilização, identificação e reconhecimento de expressões de raiva em homens do que nas mulheres, o que ajuda a explicar os TR mais rápidos perante a deteção de faces masculinas. Estes pressupostos podem ajudar a compreender o facto de no nosso estudo terem sido obtidos piores resultados ao nível da capacidade de deteção (menor proporção de respostas corretas) desta emoção perante alvos com faces de mulheres, provavelmente, por estas serem encaradas como menos ameaçadoras do que os homens (Fessler et al., 2004). Por outro lado, e relativamente ao processamento do nojo, a psicologia evolutiva explica que esta emoção (nojo) é uma resposta à possibilidade de contaminação, que resultava em comportamentos de evitamento, de modo a reduzir a exposição do indivíduo a agentes patogénicos e toxinas (e.g., Curtis, Aunger, \& Rabie, 2004; Curtis \& Biran, 2001), permitindo-nos diminuir os comportamentos de risco. Como as mulheres ancestrais estavam mais ativas para responder a riscos no domínio da reprodução e criação dos filhos, estas apresentam maiores reações de nojo que lhes permitem proteger a sua saúde e a dos seus descendentes (e.g., Fessler et al., 2004). Provavelmente, devido ao facto de os participantes do nosso estudo serem do sexo feminino, estes responderam mais rapidamente (TR mais rápidos) perante expressões faciais masculinas de nojo, pela importância das mulheres escolherem um parceiro (homem) "saudável" que lhes permita reproduzir-se sem ocorrer perigo de contaminação (protegendo assim a sua saúde e a dos seus filhos). Contudo, por a maioria dos estudos realizados nesta área não incluírem expressões faciais de nojo, é difícil especular sobre a diferenciação em termos do processamento das emoções de raiva e nojo, sendo interessante procurar estudar estas duas emoções em simultâneo em estudos futuros.

A maioria da literatura aponta para a existência de diferenças significativas entre os indivíduos com elevados níveis de alexitimia e a restante população no processamento em geral, enquanto o nosso estudo sugere que não existem diferenças ao nível da deteção, em particular. Contudo, os resultados obtidos nesta investigação são preliminares, sendo necessário realizar estudos adicionais, controlando diferentes variáveis que nos permitam obter uma melhor compreensão do problema. Nesse sentido, sugere-se que pesquisas futuras manipulem o tempo de exposição 
das matrizes de modo a avaliar o curso temporal associado à deteção das expressões faciais com diferentes conteúdos emocionais. Por outro lado, apesar de na presente investigação se ter efetuado uma rigorosa seleção dos participantes e adotado um paradigma bem validado, o da procura visual (e.g., Wolfe, 1998), utilizando estímulos previamente testados (Lundqvist et al., 1998), os nossos participantes eram apenas do sexo feminino, revelando-se pertinente no futuro utilizar uma amostra mais representativa (participantes do sexo feminino e masculino). Idealmente, seria ainda pertinente procurar incluir no grupo experimental apenas indivíduos pertencentes ao grupo clínico (grupo mais homogéneo), uma vez que esse fator poderá ter atenuado alguns dos efeitos obtidos, contribuindo para a ausência de diferenças significativas entre os grupos.

\section{REFERÊNCIAS}

Adolphs, R., Damasio, H., Tranel, D., \& Damasio, A. R. (1996). Cortical systems for the recognition of emotion in facial expressions. Journal of Neuroscience, 16, 7678-7687.

Aftanas, L., \& Varmalov, A. (2007). Effects of Alexithymia on the Activity of the Anterior and Posterior Areas of the Cortex of the Right Hemisphere in Positive and Negative Emotional Activation. Neuroscience and Behavioral Physiology, 37(1), 67-73. doi:10.1007/s11055-007-0151-z

Bagby, R. M., Parker, J. D., \& Taylor, G. J. (1994a). The Twenty-Item Toronto Alexithymia Scale-I. Item selection and cross-validation of the factor structure. Journal of Psychosomatic Research, 38(1), 23-32. doi:10.1016/0022-3999(94)90005-1

Bagby, R. M., Parker, J. D., \& Taylor, G. J. (1994b). The Twenty-Item Toronto Alexithymia Scale-II. Convergent, discriminant, and concurrent validity. Journal of Psychosomatic Research, 38(1), 33-40. doi:10.1016/0022-3999(94)90006-X

Berthoz, S., Artiges, E., Moortele, P., Poline, J., Rouquette, S., Consoli, S., \& Martinot, J. (2002). Effect of Impaired Recognition and Expression of Emotions on Frontocingulate Cortices: An fMRI Study of Men With Alexithymia. American Journal of Psychiatry, 159(6), 961-967. doi:10.1176/ appi.ajp.159.6.961

Blair, R. J., Morris, J. S., Frith, C. D., Perrett, D. I., \& Dolan, R. J. (1999). Dissociable neural responses to facial expressions of sadness and anger. Brain, 122(5), 883-893. doi:10.1093/brain/122.5.883

Carton, J. S., Kessler, E. A., \& Pape, C. L. (1999). Nonverbal decoding skills and relationship well-being in adults. Journal of Nonverbal Behavior, 23, 91-100. doi:10.1023/A:1021339410262

Chentsova-Dutton, Y., \& Tsai, J. (2007). Gender differences in emotional response among European Americans and Hmong Americans. Cognition and Emotion, 21(1), 162-181. doi:10.1080/02699930600911333

Curtis, V., Aunger, R., \& Rabie, T. (2004). Evidence that disgust evolved to protect from risk of disease. Proceedings of the Royal Society Biological Sciences Series, 271(4), 131-133. doi:10.1098/ rsbl.2003.0144

Curtis, V., \& Biran, A. (2001). Dirt, disgust, and disease: Is hygiene in our genes? Perspectives in Biology and Medicine, 44(1), 17-31. doi:10.1353/pbm.2001.0001

Darwin, C. (1998). The Origin of Species. Oxford: University Press. 
Driver, J., \& Frackowiak, R. S. (2001). Imaging selective attention in the human brain. Editora: Elsevier Amstersdam.

Fessler, D. M., Pillsworth, E. G., \& Flamson, T. J. (2004). Angry men and disgusted women: An evolutionary approach to the influence of emotions on risk taking. Organizational Behavior and Human Decision Processes, 95, 107-123. doi:10.1016/j.obhdp.2004.06.006

Franz, M., Schaefer, R., Schneider, C., Sitte, W., \& Bachor, J. (2004). Visual event-related potentials in subjects with alexithymia: modified processing of emotional aversive information? American Journal Psychiatry, 161(4), 728-35.

Galera, C. A. (2003). Stimuli selection and attentive analysis in the visual search task: Evidences of discrete and sequential stages. Arquivo Brasileiro de Oftalmologia, 66, 114-120.

Goeleven, E., De Raedt, R., Leyman, L., \& Verschuere, B. (2008). The Karolinska Directed Emotional Faces: A validation study. Cognition \& Emotion, 22(6), 1094-1118. doi10.1080/02699930701626582

Hansen, C. H., \& Hansen, R. D. (1988). Finding the face in the crowd: An anger superiority effect. Journal of Personality and Social Psychology, 54, 917-924. doi:10.1037/0022-3514.54.6.917

Humphreys, T. P., Wood, L. M., \& Parker, J. D. (2009). Alexithymia and satisfaction in intimate relationships. Personality and Individual Differences, 46, 43-47. doi:10.1016/j.paid.2008.09.002

Izard, C. E. (1991). The Psychology of Emotions. Plenum Press, New York.

Jessimer, M., \& Markham, R. (1997). Alexithymia: A Right Hemisphere Dysfunction Specific to Recognition of Certain Facial Expressions? Brain and Cognition, 34, 246-258. doi:10.1006/ brcg.1997.0900

Kano, M., Fukudo, S., Gyoba, J., Kamachi, M., Tagawa, M., Mochizuki, H., ... Yanai, K. (2003). Specific brain processing of facial expressions in people with alexithymia: an H2 15O-PET study. Brain, 126, 1474-1484. doi:10.1093/brain/awg131

Karlsson, H., Näätänen, P., \& Stenman, H. (2008). Cortical activation in alexithymia as a response to emotional stimuli. The British Journal of Psychiatry, 192, 32-38. doi:10.1192/bjp.bp.106.034728

Keltner, D., Ekman, P., Gonzaga, G. C., \& Beer, J. (2003). Facial expression of emotion. In R. J. Davidson, K. R. Scherer, \& H. H. Goldsmith (Eds.), Handbook of affective sciences (pp. 415-432). New York, NY: Oxford University Press.

Kugel, H., Eichmann, M., Dannlowski, U., Ohrmann, P., Bauer, J., Arolt, V., ... Suslow, T. (2008). Alexithymic features and automatic amygdala reactivity to facial emotion. Neuroscience Letters, 435, 40-44. doi:10.1016/j.neulet.2008.02.005

Lane, R. D., Sechrest, L., Reidel, R., Shapiro, D. E., \& Kaszniak, A. W. (2000). Pervasive emotion recognition deficit common to alexithymia and repressive coping style. Psychosomatic Medicine, 62, 492-501.

Lane, R. D., Sechrest, L., Reidel, R., Weldon, V., Kaszniak, A., \& Schwartz, G. E. (1996). Impaired verbal and nonverbal emotion recognition in alexithymia. Psychosomatic Medicine, 58, 203-10.

Lazarus, R. S. (1991). Emotion and adaptation. New York, NY: Oxford University Press.

Luminet, O., Rimé, B., Bagby, R. M., \& Taylor, G. J. (2004). A multimodal investigation of emotional responding in alexithymia. Cognition and Emotion, 18 (6), 741-766. doi:10.1080/02699930341000275

Lundqvist, D., Flykt, A., \& Öhman, A. (1998). The Karolinska Directed Emotional Faces - KDEF [CDROM]. Stocholm: Karolinska Institutet, Department of Clinical Neuroscience, Psychology section.

Mantani, T., Okamoto, Y., Shirao, N., Okada, G., \& Yamawaki, S. (2005). Reduced activation of posterior cingulate cortex during imagery in subjects with high degrees of alexithymia: a functional magnetic resonance imaging study. Biological Psychiatry, 57(9), 982-990. doi:10.1016/j. biopsych.2005.01.047 
Mayer, J. D., DiPaolo, M., \& Salovey, P. (1990). Perceiving affective content in ambiguous visual stimuli: A component of emotional intelligence. Journal of Personality Assessment, 54, 772-307.

Mériau, K., Wartenburger, I., Kazzer, P., Prehn, K., Lammers, C., Meer, E., Villringer, A., \& Heekeren, H. (2006). A neural network reflecting individual differences in cognitive processing of emotions during perceptual decision making. Neuroimage, 33, 1016-1027. doi:10.1016/j.neuroimage.2006.07.031

Moriguchi, Y., Ohnishi, T., Lane, R., Maeda, M., Mori, T., Nemoto, K., ... Komaki, G.(2006). Impaired self-awareness and theory of mind: An fMRI study of mentalizing in alexithymia, Neuroimage 32, 1472-1482. doi:10.1016/j.neuroimage.2006.04.186

Nemiah, J. C., Freyberger, H., \& Sifneos, P. E. (1976) Alexithymia: a view of the psychosomatic process. In: J. Hillow (Ed), Modern trends in psychosomatic medicine. (pp. 430-439). London: Butterworths.

Oatley, K., \& Jenkins, J. M. (1996). Understanding Emotions. London: Blackwell.

Öhman, A. (1986). Face the beast and fear the face: animal and social fears as prototypes for evolutionary analyses of emotion. Psychophysiology, 23, 123-145.

Öhman, A., Juth, P., \& Lundqvist, D. (2010). Finding the face in a crowd: Relationships between distractor redundancy, target emotion, and target gender. Cognition \& Emotion. 24(7), 12161228. doi: $10.1080 / 02699930903166882$

Öhman, A., Lundqvist, D., \& Esteves, F. (2001). The face in the crowd revisited: A threat advantage with schematic stimuli. Journal of Personality and Social Psychology, 80, 381-396. doi:10.1037/00223514.80.3.381

Parker, P., Prkachin, K., \& Prkachin, G. (2005). Processing of Facial Expressions of Negative Emotion in Alexithymia: The Influence of Temporal Constraint. Journal of Personality 73(4), doi:10.1111/j.1467-6494.2005.00339.x

Parker, J. D., Taylor, G. J., Bagby, R. M., \& Acklin, M. W. (1993). Alexithymia in panic disorder and simple phobia: A comparative study. American Journal Psychiatry, 150, 1106-1109.

Pashler, H., Dobkins, K., \& Huang, L. (2004). Is contrast just another feature for visual selective attention? Vision Research, 44, 1403-1410. doi:10.1016/j.visres.2003.11.025

Phan, K. L., Wager, T., Taylor, S. F., \& Liberzon, I. (2002). Functional neuroanatomy of emotion: a meta-analysis of emotion activation studies in PET and fMRI. Neuroimage, 16, 331-348. doi:10.1006/nimg.2002.1087

Prazeres, N., Parker, D., \& Taylor, J. (2000). Adaptação Portuguesa da Escala de Alexitimia de Toronto de 20 Itens (tas-20). RIDEP, 9(1), 9-21

Prkachin, G. C., Casey, C., Prkachin, K. M. (2009). Alexithymia and perception of facial expressions of emotion. Personality and Individual Differences, 46, 412-417

Prkachin, G. C., \& Prkachin, K. M. (2001). Alexithymia and detection of facial expressions of emotion. Psychosomatic Medicine, 63, 135-136. doi:10.1016/j.paid.2008.11.010

Rauschenberger, R., \& Yantis, S. (2006). Perceptual encoding efficiency in visual search. Journal of Experimental Psychology, 135, 116-131. doi:10.1037/0096-3445.135.1.116

Reker, M., Ohrmanna, P., Raucha, A., Kugelb, H., Bauera, J., Dannlowskia, U., ... Suslowa, T. (2010). Individual differences in alexithymia and brain response to masked emotion faces. Cortex, 46, 658-667. doi:10.1016/j.cortex.2009.05.008

Scherer, K. R. (1984). On the nature and function of emotion: A component process approach. In K. R. Scherer \& P. Ekman (Eds.), Approaches to emotion, pp. 293-318. Hillsdale, NJ: Erlbaum.

Schneider, W., Eschman, A., \& Zuccolotto, A. (2002). E-Prime user's guide. Pittsburgh, PA: Psychology Software Tools. 
Schwarz, N. (1990). Feelings as information: Informational and motivational functions of affective states. In E. T. Higgins \& R. M. Sorrentino (Eds.), Handbook of motivation and cognition: Foundations of social behavior (pp. 527-561). New York: Guilford Press.

Silva, A., \& Vasco, A. (2010). Alexitimia: Que processos emocionais? Que intervenção terapêutica? Actas do VII Simpósio Nacional de Investigação em Psicologia, Universidade do Minho, Portugal.

Sroufe, L. A. (1996). Emotional development: The organization of emotional life in the early years. New York, NY: Cambridge University Press.

Stratton, P., \& Hayes, N. (1999). A student's dictionary of psychology. New York, NY: Arnold

Taylor, G. J., \& Bagby, R. M. (2004). New trends in alexithymia research. Psychother and Psychosom, 73, 68-77. doi: 10.1159/000075537

Taylor, G. J., Bagby, R. M., \& Parker, D. A. (1997). Disorders of affect regulation: alexithymia in medical and psychiatric illness. New York, NY: Cambridge University Press.

Taylor, G. J., Ryan, D., \& Bagby, R. M. (1985). Toward the development of a new self-report alexithymia scale. Psychotherapy and Psychosomatics, 44, 191-199.

Vanheule, S., Vandenbergen, J., Verhaeghe, P., \& Desmet, M. (2010). Interpersonal problems in alexithymia: A study in three primary care groups. Psychology and Psychotherapy: Theory, Research and Practice, 83, 351-362. doi:10.1348/147608309X481829

Veríssimo, Ramiro (2001). Versão portuguesa da Escala de Alexitimia de Toronto de 20 itens. Acta Médica Portuguesa, 14, 529-536.

Vermeulen, N., Luminet, O., \& Corneille, O. (2006). Alexithymia and the automatic processing of affective information: Evidence from the affective priming paradigm. Cognition and emotion, 20(1), 64-91. doi:10.1080/02699930500304654

Vermeulen, N., Luminet, O., Sousa, M., \& Campanella, S. (2008). Categorical perception of anger is disrupted in alexithymia: Evidence from a visual ERP study. Cognition and Emotion, 22, 1052-1067. doi:10.1080/02699930701597635

Wolfe, J. M. (1998). Visual search. In H. Pashler (Ed.), Attention (pp. 13-73). Hove, UK: Psychology Press. 


\section{FIGURAS}
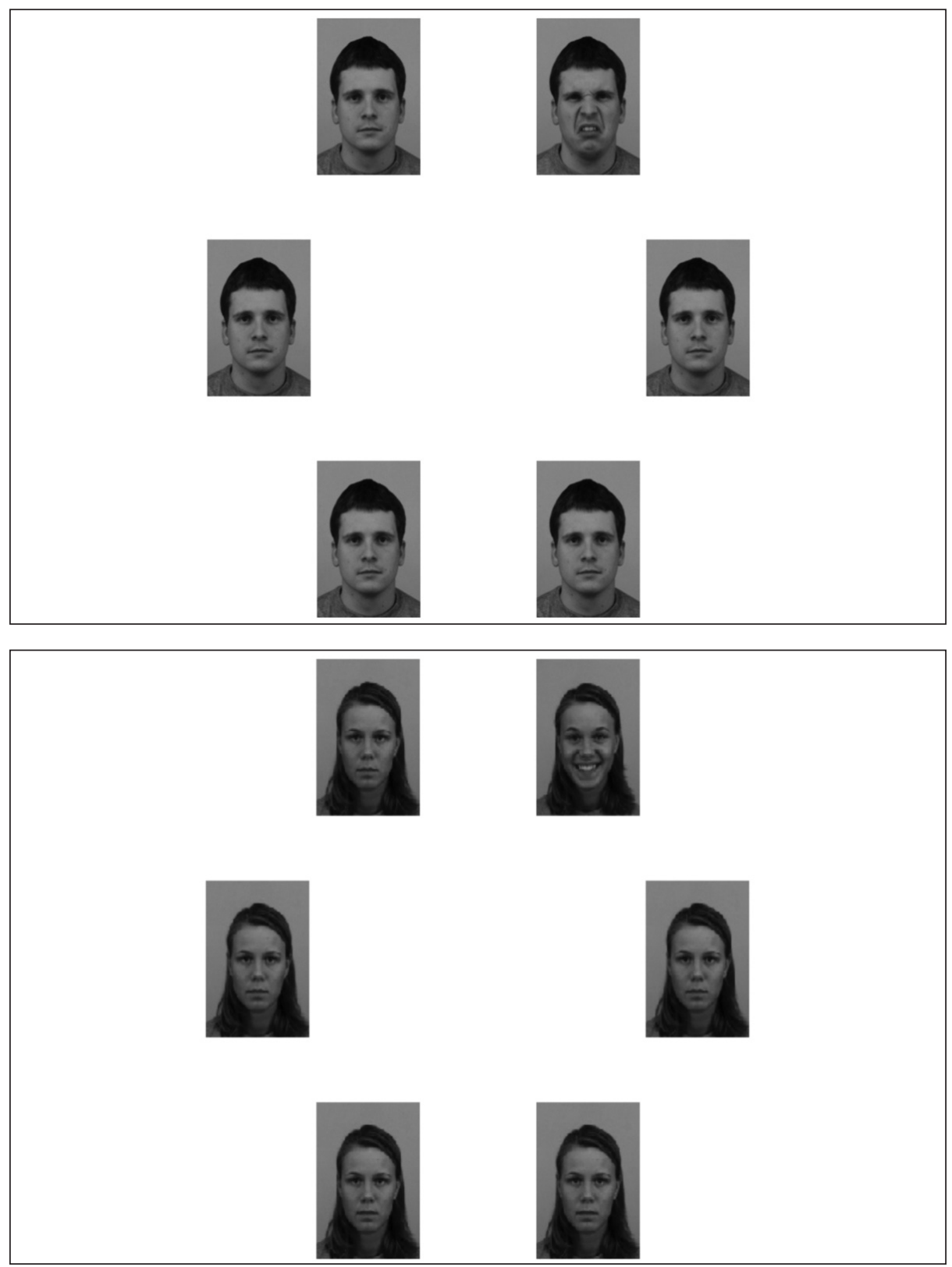

Figura 1

Exemplo de duas matrizes, uma de faces masculinas e outra de faces femininas, com alvos discrepantes (respetivamente, nojo e alegria), rodeadas de distratores (expressões emocionais neutras). 


\section{Tempo de Resposta}
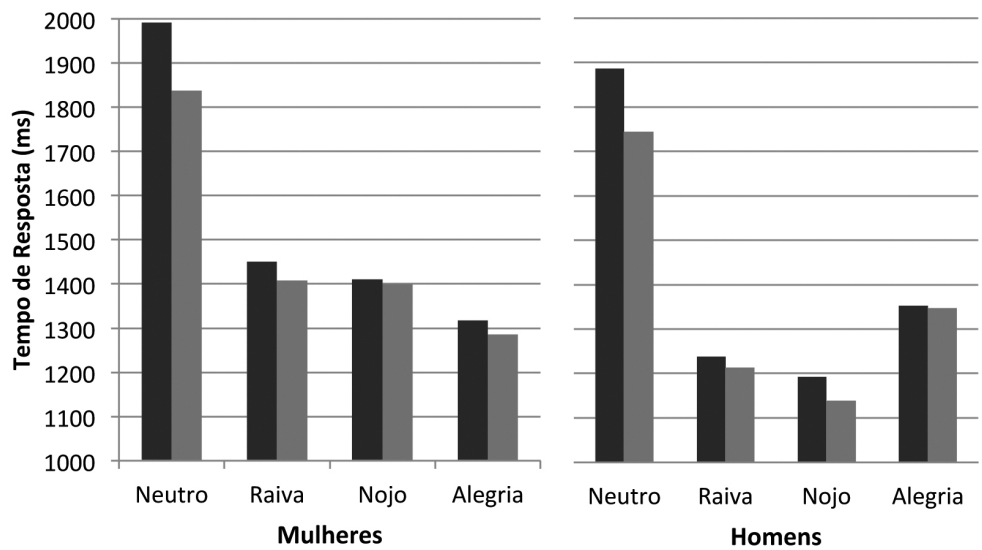

- Baixos níveis de alexitimia

Elevados níveis de alexitimia

Figura 2

Os gráficos apresentam os TR dos dois grupos (baixos e elevados níveis de alexitimia) ao nível da deteção dos diferentes alvos (faces com expressões neutras, raiva, alegria e nojo) em faces de mulheres e homens, respetivamente

\section{Tempo de Resposta}

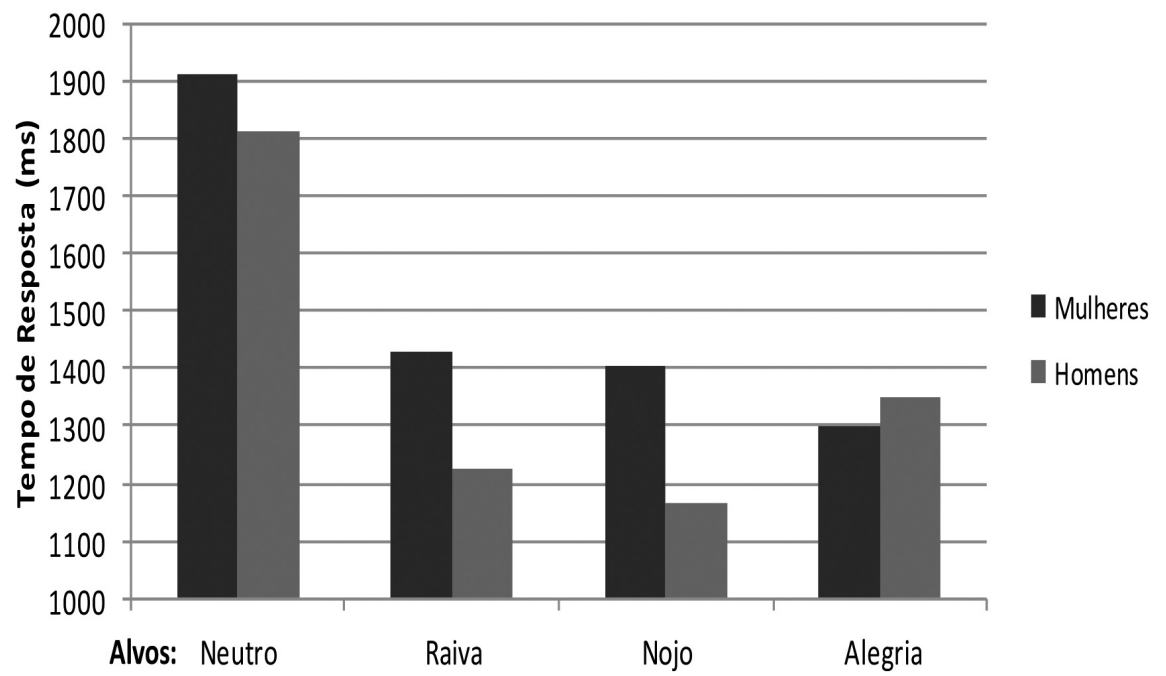

Figura 3

Interação entre o género (feminino e masculino) e as diferentes expressões faciais (neutra, raiva, nojo e alegria) ao nível dos TR. 


\section{Proporção de Respostas Corretas}


Figura 4

Os gráficos apresentam a proporção de respostas corretas obtidas pelos dois grupos (baixos e elevados níveis de alexitimia) ao nível da deteção dos diferentes alvos (faces com expressões neutras, raiva, alegria e nojo) em faces de mulheres e homens, respetivamente.

\section{Proporção de Respostas Corretas}

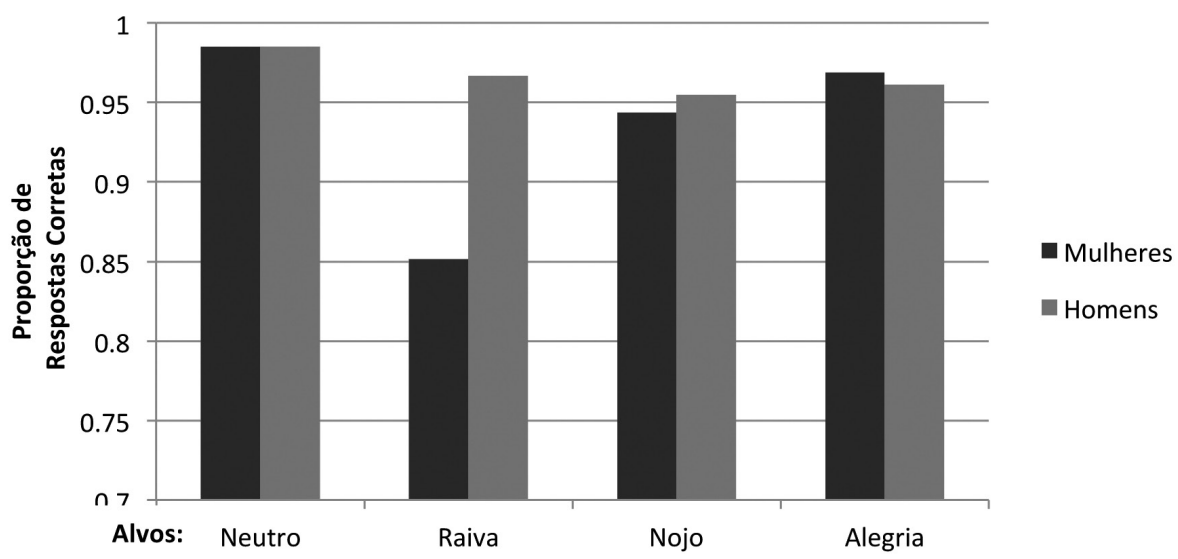

Figura 5

Interação entre o género (feminino e masculino) e as diferentes expressões faciais (neutra, raiva, nojo e alegria) ao nível da proporção de respostas corretas. 